\title{
Gonorrhoe in Grossbritannien, 1967
}

British Cooperative Clinical Group

Die Ergebnisse von 186 Kliniken wurden zusammengefasst und bein-halten über 90 \% der Erkrankungen. In England und Wales werden 1622, in Schottland 843 weibliche Erkrankte gemeldet. Bei den Männern sind die Zahlen 2724 und 2092. Die Infektionen haben in den letzten Jahren ständig zugenommen. Es ist eine Ballung der Infektionshäufigkeit in den grossen Städten festzustellen. Das Verhältnis von Frauen zu Männern ist zurzeit 1:2,8 in England und Wales bzw. 1:2,5 in Schottland. Bei Ver-gleichen mit früheren Analysen zeigt sich, dass der Anteil der infizierten Frauen ständig grosser wird.

Bei Aufgliederung der Erkrankungszahlen nach den einzelnen Rassen sieht man, dass in Kleinstädten hauptsächlich in Grossbritannien Gebo-rene infiziert werden, während in Grossstädten der Anteil der Einwande-rer sehr gross ist. Dieser Trend ist bei den Frauen noch starker als bei den Männern. In Schottland waren nicht weniger als 96,5 \% der Frauen in Grossbritannien geboren; in England und Wales 82,1 \%. 6,9 \% der Patientinnen stammten aus Westindien und alle übrigen Emigrantinnen betrugen 10,9\%, davon Irinnen 4,1 und Europäerinnen 2,6 \%. Aber nur 12 Frauen aus Westindien und 34 andere Emigrantinnen wurden in Städten unter 100000 Einwohnern behandelt.

1 Original tit el: Gonorrhoea study, 1967.

190

\section{British Cooperative Clinical Group}

Bei den Männern liegen die Prozentzahlen etwas anders. Sie seien zum Vergleich angeführt: in Schottland 87,8 \%, in England und Wales 56,4 \%. In England und Wales, der Gegend der Grossstädte waren 17,3 \% der In-fizierten aus Westindien und 26,3 \% übrige Emigranten. Unter ihnen stel-Ien die Asiaten den grössten Anteil mit 7,5 \%, gefolgt von Negern, da in Afrika die Gonorrhoe in einigen Gebieten epidemisch auftritt.

Ein Vergleich mit früheren Studien aus England und Wales, Manner und Frauen betreffend, zeigt, dass der Anteil der erkrankten Briten stän-dig leicht steigt, während die Zahl der erkrankten Einwanderer aus Westindien abnimmt. Der Anteil der übrigen Emigrantengruppen bleibt etwa gleich. Die Gesamtzunahme an Infektionen scheint vor allem durch die höhere Infektionsquote bei den Briten zu entstehen.

Das Verhältnis der erkrankten Frauen zu den Männern, aufgeschlüs-selt nach der Rasse, ergibt folgende Werte: Westindien 1 Frau auf 6,9 Manner; andere Emigranten 1:6,7 und Briten 1:1,9. Der Anteil an infizierten Männern ist also unter den Emigranten um vieles grosser als unter der autochthonen Bevölkerung. Im Anhang wird eine Untersuchung gezeigt, die die Behandlung durch private Ärzte in Schottland ergab. Nur ein ganz geringer Teil der Privatärzte behandelt die Patienten selbst; es werden fast alle in Kliniken des Gesundheitsdienstes geschickt. Dies wird als Beweis dafür gewertet, dass die Ergebnisse der umfassenden Untersuchung, die sich auf Ergebnisse von Spitälern des Gesundheitsdienstes stützt, den tatsächlichen Verhältnissen entsprechen. H.Stöger 